\title{
THE K500-to-K1200 COUPLING LINE FOR THE COUPLED CYCLOTRON FACILITY AT THE NSCL*
}

\author{
X. Wu, H. Blosser, D. Johnson, F. Marti, R.C. York \\ National Superconducting Cyclotron Laboratory \\ Michigan State University \\ East Lansing, MI 48824
}

\begin{abstract}
An upgrade now in progress at the National Superconducting Cyclotron Laboratory (NSCL) facility will couple two existing superconducting cyclotrons. This will provide significant beam intensity gains for all ions and for heavier ions, also increased energy. These gains will greatly enhance the performance of the NSCL facility, particularly for nuclear studies with radioactive beams and nuclear reaction physics. A significant element of this upgrade is the K500-to-K1200 coupling line used to transport beam between the two cyclotrons. Beyond satisfying the geometric constraints of beam transport, the system must match the six-dimensional phase space obtained from the K500 to that required for injection into the K1200. The transported beams range from low energy, heavy ions like ${ }^{238} \mathrm{U}^{28+}$ at $6.9 \mathrm{MeV} / \mathrm{u}$ to high energy, light ions like ${ }^{16} \mathrm{O}^{3+}$ at $16.7 \mathrm{MeV} / \mathrm{u}$. Tracking studies have been done to simulate the required matching conditions and performance of the coupling line. The result of these studies, including required magnets and beam diagnostics are presented in this paper.
\end{abstract}

\section{INTRODUCTION}

The coupled cyclotron facility at the NSCL will consist of an ECR-ion-source-injected K500 cyclotron followed by a K500-to-K1200 coupling line, and then by radial, chargestriping injection into the K1200 cyclotron for final acceleration to 100-200 MeV/nucleon [1]. The basic requirement for the K500-to K1200 coupling line is to transport the required ion beams between two cyclotrons and match the six-dimensional phase space to ensure efficient injection into the K1200 cyclotron. A wide range of ion beams is required to support the proposed nuclear physics research program. Table 1 shows a selected beam list for the K500-to-K1200 coupling line. For this analysis, the beam emittance from the K500 cyclotron was assumed to be $5 \pi \mathrm{mm}$-mrad (unnormalized) with a momentum spread $\Delta \mathrm{p} / \mathrm{p}$ of $0.2 \%$. The required ion beams for the K500-to-K1200 coupling line will have a maximum beam momentum P/Q of $1.0 \mathrm{GeV} / \mathrm{c}$ and a charge/mass ratio $\mathrm{Q} / \mathrm{A}<0.2$.

"Work supported by NSF contract number PHY952884.
Table 1: Beam List for the K500-to-K1200 Coupling Line

\begin{tabular}{|c|c|c|c|}
\hline ION & A & Q & $\begin{array}{c}\text { Energy } \\
(\mathrm{MeV} / \mathrm{u})\end{array}$ \\
\hline $\mathrm{O}$ & 16 & 3 & 16.7 \\
\hline $\mathrm{Ar}$ & 40 & 7 & 15.3 \\
\hline $\mathrm{Kr}$ & 84 & 14 & 14.0 \\
\hline $\mathrm{Xe}$ & 129 & 21 & 12.9 \\
\hline $\mathrm{U}$ & 238 & 28 & 6.9 \\
\hline
\end{tabular}

\section{COUPLING LINE MATCHING CONDITIONS}

The matching conditions for the coupling were obtained by simulating the K500 beam extraction and the K1200 beam injection process.

The K500 beam extraction simulation was done using magnetic field measurements both in the acceleration and extraction regions [2]. The ion beams were tracked using code CYCLONE from the spiral inflector at the center, through the central region electrodes, accelerating region and K500 extraction system to the beginning of the coupling line. The modified K500 extraction system consists of 2 electrostatic deflectors and 7 focusing bars. In addition, a new combined function magnet was implemented to provide additional deflection and focusing. The design philosophy was to maintain the beam envelope within the available aperture and to minimize the divergence of the beam at the exit of the cyclotron. To obtain this objective not all bars are active for each test beam. The resultant K500 extracted beam parameters for 3 typical beams at the matching point are given in Table 2 .

The K1200 injection simulation [3] was done by backward tracking of particle trajectories from the striping foil inside K1200 (at a radius of $\sim 33 \mathrm{~cm}$ ) through the Dee gap and injection channel of the yoke towards the end of the coupling line. A new combined-function magnet inside the injection channel is required to provide both beam deflection and focus. The resultant K1200 injected beam parameters for the same 3 beams at the end of the coupling line are given in Table 3. 


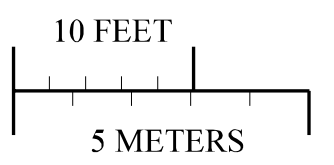

RTECR

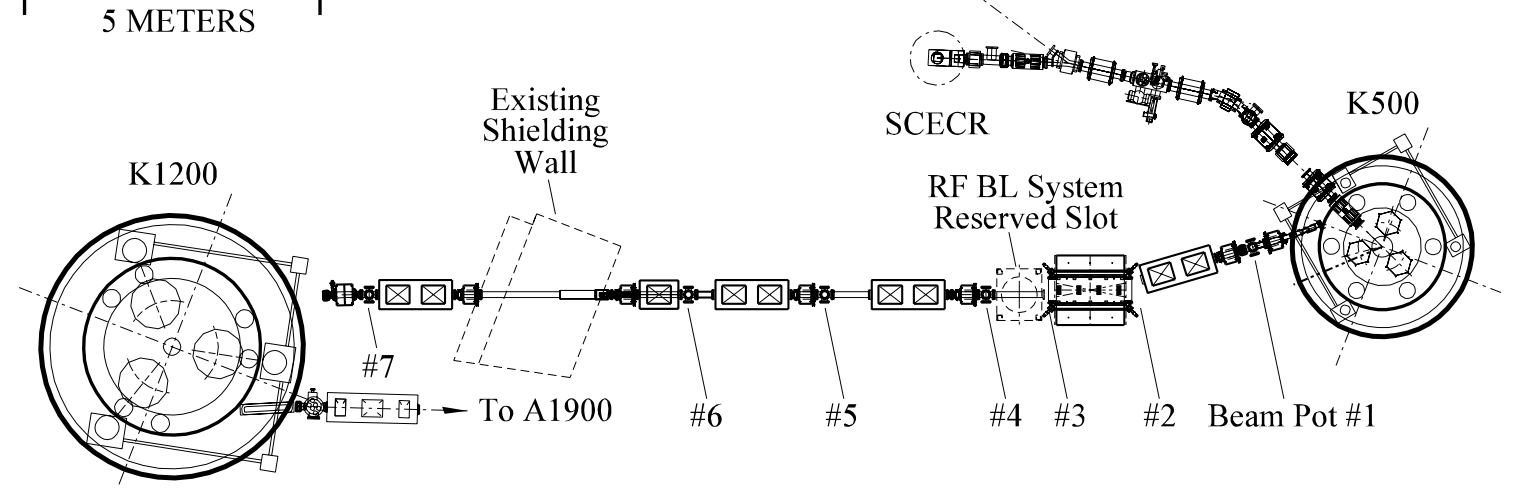

Figure 1. Layout of the proposed K500-to-K1200 coupling line.

Table 2: K500 Extracted Beam Parameters.

\begin{tabular}{|c|c|c|c|}
\hline $\begin{array}{c}\text { Beam } \\
\text { Parameter }\end{array}$ & ${ }^{16} \mathbf{O}^{3+}$ & ${ }^{40} \mathbf{A r}^{6+}$ & ${ }^{{ }^{238}} \mathbf{U}^{21+}$ \\
\hline$\beta_{x}(\mathrm{~m})$ & 4.723 & 2.999 & 3.663 \\
\hline$\alpha_{\mathrm{x}}$ & -2.517 & -2.442 & -2.588 \\
\hline$\beta_{\mathrm{v}}(\mathrm{m})$ & 0.918 & 1.135 & 0.482 \\
\hline$\alpha_{\mathrm{v}}$ & 0.429 & 0.494 & 0.052 \\
\hline$\eta_{\mathrm{x}}(\mathrm{m})$ & 4.488 & 5.563 & 5.327 \\
\hline$\eta_{\mathrm{x}}{ }^{2}$ & 1.440 & 1.809 & 2.784 \\
\hline
\end{tabular}

Table 3: K1200 Injected Beam Parameters.

\begin{tabular}{|c|c|c|c|}
\hline $\begin{array}{c}\text { Beam } \\
\text { Parameter }\end{array}$ & ${ }^{16} \mathbf{O}^{3+}$ & ${ }^{40} \mathbf{A r}^{6+}$ & ${ }^{238} \mathbf{U}^{21+}$ \\
\hline$\beta_{x}(\mathrm{~m})$ & 121.274 & 86.946 & 49.251 \\
\hline$\alpha_{x}$ & -30.195 & -34.733 & -21.839 \\
\hline$\beta_{\mathrm{y}}(\mathrm{m})$ & 3.990 & 5.794 & 12.846 \\
\hline$\alpha_{\mathrm{y}}$ & 2.012 & 3.233 & 7.838 \\
\hline$\eta_{\mathrm{x}}(\mathrm{m})$ & 5.770 & 5.050 & 4.440 \\
\hline$\eta_{\mathrm{x}}{ }$ & 1.720 & 2.279 & 2.279 \\
\hline
\end{tabular}

\section{K500-TO-K1200 COUPLING LINE}

Figure 1 shows the configuration of the planned, K500to-K1200 coupling line. The lattice uses a single $16^{\circ}$ dipole, a quadrupole singlet, and four quadrupole doublets to transport the beam from K500 to K1200 while satisfying the required matching conditions. All of these magnets will be obtained from the decommissioned A1200 fragment separator. The dipole has a full gap of $5.0 \mathrm{~cm}$. The quadrupoles have an effective length of $0.4 \mathrm{~m}$ and a radial aperture of 6.5 $\mathrm{cm}$. The maximum quadrupole field gradient available is about $35 \mathrm{~T} / \mathrm{m}$. A slot has also been reserved for a RF system for possible future control of the bunch length between the two cyclotrons.

The resultant coupling line $\beta$ functions for the 3 beams covering the operating diagram are shown in Figure 2, 3 and 4, respectively with the dispersion functions shown in Figure 5. Due to the varied matching conditions required for different beams, the tuning of the coupling line changes significantly. Beam simulations were done to check whether the coupling line could match K1200 beam conditions if the K500 beam conditions varied from $50 \%$ to $200 \%$ of the original value. The results were very satisfactory. In addition, as shown in Figure 5, the coupling line was designed to achieve $\eta=0$ at the same position for all beams where a 4-jaw collimator will be placed to clean up the phase space from the K500.

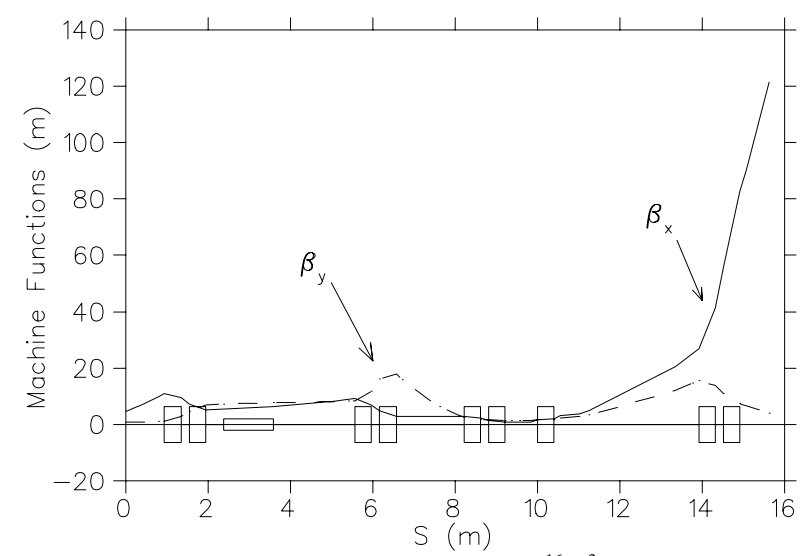

Figure 2. Lattice functions for ${ }^{16} \mathrm{O}^{3+}$ beam 


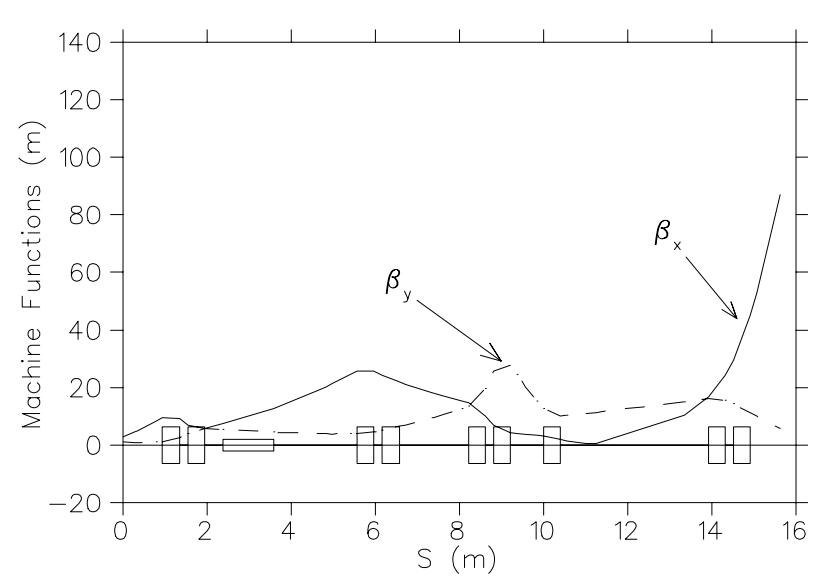

Figure 3. Lattice functions for ${ }^{40} \mathrm{Ar}^{6+}$ beam.

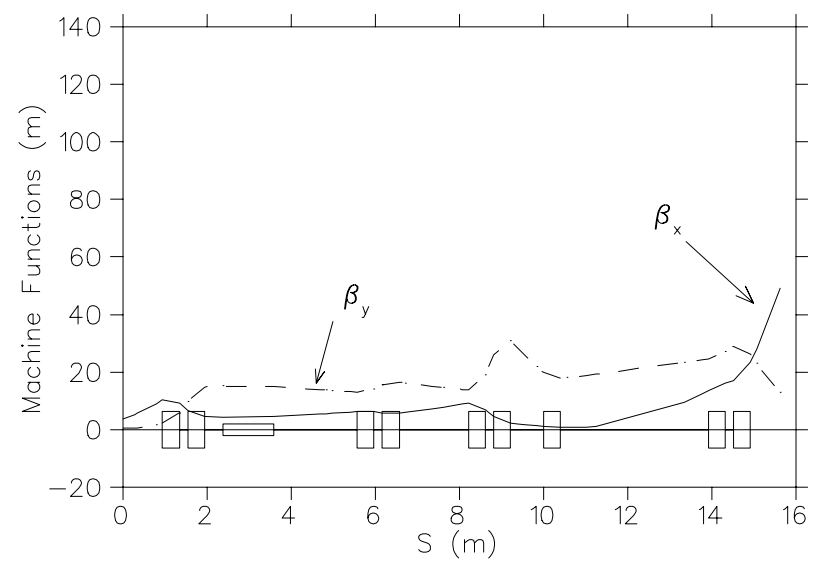

Figure 4. Lattice functions for ${ }^{238} \mathrm{U}^{21+}$ beam.

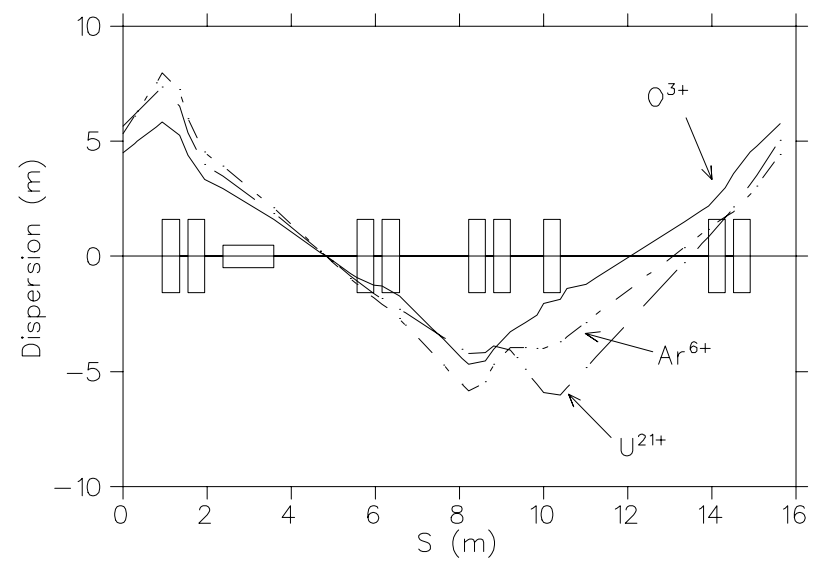

Figure 5. Dispersion functions for all 3 beams.

The beam envelopes for all 3 beams with an emittance of $5.0 \pi \mathrm{mm}-\mathrm{mrad}$ and a momentum spread $\Delta \mathrm{p} / \mathrm{p}$ of $0.2 \%$ are shown in Figure 6, and the maximum required quadrupole gradients are listed in Table 4 . The beam envelopes are well within in the aperture limits and the field gradient requirements can easily be achieved.

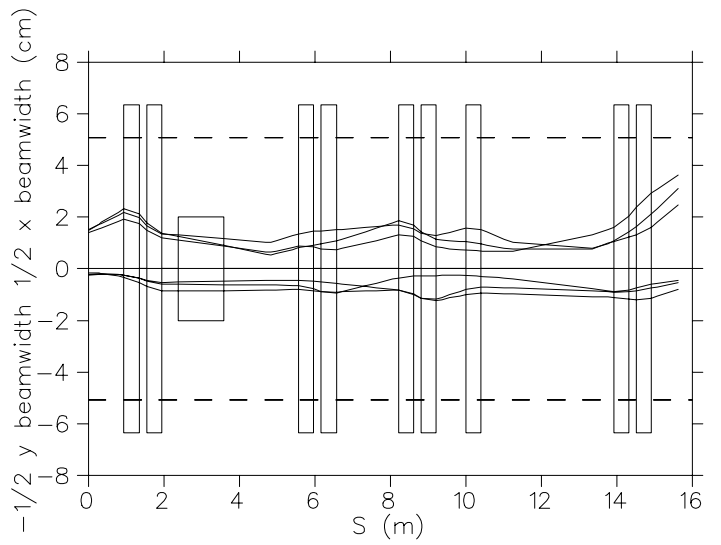

Figure 6. Beam envelopes for all 3 beams.

Table 4: Required Coupling Line Quadrupole Gradient.

\begin{tabular}{|c|c|c|c|}
\hline \multirow{2}{*}{ Quadrupole } & \multicolumn{3}{|c|}{ Quadruple Gradient (T/m) } \\
\cline { 2 - 4 } & ${ }^{16} \mathbf{O}^{3+}$ & ${ }^{40} \mathbf{A r}^{6+}$ & ${ }^{238} \mathbf{U}^{\mathbf{2 1 +}}$ \\
\hline QK5F1 & 7.19 & 7.03 & 7.47 \\
\hline QK5F2 & -4.50 & -5.19 & -6.18 \\
\hline QK12F1 & 9.19 & -1.02 & 6.81 \\
\hline QK12F2 & -7.87 & 0.71 & -4.60 \\
\hline QK12F3 & 6.63 & 6.38 & -0.31 \\
\hline QK12F4 & -4.87 & -5.92 & -1.86 \\
\hline QK12F5 & 1.51 & 1.44 & 5.03 \\
\hline QK12F6 & -2.00 & -0.82 & 2.54 \\
\hline QK12F7 & -0.28 & -1.86 & -5.10 \\
\hline
\end{tabular}

\section{BEAM DIAGNOSTICS}

The K500-to-K1200 coupling line tuning depends closely on the K500 beam conditions. The determination of beam emittance, phase space parameters, beam energy and energy spread from the K500 is very important for proper K1200 striping injection. As shown in Figure 1, the coupling line will have 7 beam diagnostic areas. Faraday cups and phosphor screens will be used to monitor the beam intensity and profile, and two non-intercepting bunch length probes at the entrance and the exit will be used to monitor beam bunch length. Beam phase space parameters will be determined with slits and a phosphor screen. In addition, we are evaluating the use of PPAC or MCP detectors for beam phase space as well as beam energy and energy spread measurements.

\section{REFERENCES}

[1] R. C. York et al., "Proposed Upgrade of the NSCL", IEEE Particle Accelerator Conference, Dallas, TX, p. 345, (1998).

[2] F. Marti et al., "Modifications to K500 focusing bars", December 1998, MSU, NSCL-CCP-19-1998.

[3]D. A Johnson et al., "Survey of injected beam parameters at the K1200 cyclotron", August 1995, NSCL-CCP6-1995. 